\title{
ON FOURIER COEFFICIENTS OF A CONTINUOUS PERIODIC FUNCTION OF BOUNDED ENTROPY NORM
}

\author{
ROMUALD DABROWSKI
}

In 1983 B. Korenblum [5, 6] introduced a new class of Banach function spaces associated with the notion of entropy (we will call these spaces and their norms entropy spaces and entropy norms, respectively). The entropy norms are intermediate between the uniform and the variation norms. One application of entropy spaces is a new convergence test for Fourier series which includes classical tests of Dirichlet-Jordan and Dini-Lipschitz [6]. The aim of this note is to point out a natural connection between entropy spaces and Hardy space $\operatorname{Re} H^{1}$ [4]. In fact any entropy space can be embedded into $\operatorname{Re} H^{1}$ via a multiplier type bounded operator. As a corollary we obtain a growth condition for Fourier coefficients of a continuous periodic function of bounded entropy norm.

1. Two representations of an entropy norm. Let $T=R / Z$ be the unit circle, and let $|E|=\int_{E} d x$ denote the normalized Lebesgue measure of a Borel subset $E$ of $T$. Also, let $k(s), 0<s \leq 1$, be a positive nondecreasing concave function such that $k(s)=1$. The $k$-entropy of a finite subset $E$ of $T(E \neq \varnothing)$ is $k(E)=\sum_{i} k\left(\left|I_{i}\right|\right)$, where $\left\{I_{i}\right\}$ are the complementary arcs of $E$ in $T$. For an infinite subset $E$ of $T$ we set $k(E)=\sup \{k(F) ; F \subset E$, $F$ finite $\}$. We also put $k(\varnothing)=0$. The $k$-entropy norm $[\mathbf{5}, 6]$ of a real continuous function $f$ on $T$ is defined by the formula

$$
\|f\|_{k}=\int_{R} k\left(f^{-1}(\{y\}) d y .\right.
$$

THEOREM 1 [2]. Let $k(s), 0<s \leq 1$, be a positive nondecreasing concave function such that $k\left(0^{+}\right)=0$ and $\lim _{s \rightarrow 0}(k(s) / s)=\infty$. There is a unique Borel probability measure $\mu_{k}$ on the unit interval $(0,1]$ such that

$$
\|f\|_{k}=\int_{T} \int_{(0,1]} \frac{1}{s} \Omega_{I}(f) d x d \mu_{k}(s),
$$

where $\Omega_{I}(f)$ is the oscillation of the function $f$ on the arc $I=I(x, s)$ in $T$ of length $s$ and center at $x$. A relationship between $k$ and $\mu_{k}$ is given by the formula

$$
k(s)=\int_{0}^{s} \int_{t}^{1} \frac{1}{u} d \mu_{k}(u) d t .
$$

It is then proved in [2] that the set $C_{k}$ of real continuous functions on $T$ of finite $k$-entropy norm is a Banach algebra with respect to the norm $\|\cdot\|_{k}+\|\cdot\|_{\infty}$.

Received by the editors February 9, 1987.

1980 Mathematics Subject Classification (1985 Revision). Primary 42A20, 46E15. 
2. Natural embedding of the space $C_{k}$ into Hardy space $\operatorname{Re} H^{1}$. We recall that a real function $a(t)$ on $T$ is called an atom if (i) the support of $a(t)$ is contained in a subarc $I$ of $T$; (ii) $|a(t)| \leq|I|^{-1}$ for all $t$ in $T$; (iii) $\int_{T} a(t) d t=0$. It is proved in [1] that

$$
\sup \left\{\|a\|_{\operatorname{Re} H^{1}}, a \text { atom }\right\}=1,
$$

for a suitable choice of norm of $\operatorname{Re} H^{1}$. From now on we fix a function $k$ with the properties assumed in Theorem 1 and we let $\mu=\mu_{k}$ be the corresponding probability measure. Also, let $\Delta_{I}$ denote the characteristic function of a subarc $I$ of $T$. For any function $f, f \in C_{k}$, we put

$$
T_{k} f(t)=\int_{T} \int_{(0,1]}\left(1 / s^{2}\right) \Delta_{I}(t)(f(t)-f(I)) d \mu_{k}(s) d x
$$

where $I=I(x, s), x \in T, 0<s \leq 1$, and $f(I)=\frac{1}{s} \int_{I} f(x) d x$. A standard argument involving Fubini's theorem shows that integral (2.2) makes sense for almost all $t$ in $T$ and it defines a bounded linear operator from the space $C_{k}$ to the space $L^{1}(T)$ of all Lebesgue integrable functions on $T$. In fact much more can be said about the operator $T_{k}$. We first observe that for any arc $I=I(x, s), x \in T, 0<s \leq 1$, one can write

$$
\frac{1}{s} \Delta_{I}(t)(f(t)-f(I))=\Omega_{I}(f) a_{I}(t), \quad t \in T,
$$

where $a_{I}(t)$ is an atom supported on $I$ and dependent on $f$. Hence (2.2) can be viewed as a Bochner integral [7] of a strongly measurable function

$$
(x, s) \mapsto \frac{1}{s} \Omega_{I(x, s)}(f) a_{I(x, s)},
$$

defined on measure space $\left(T \times(0,1], d x d \mu_{k}\right)$ with values in $\operatorname{Re} H^{1}$. Now the Minkowski's inequality for the norm of a Bochner integral and (2.1) yield the following result.

THEOREM 2. $T_{k}$ is a bounded linear operator from space $C_{k}$ to Hardy space $\operatorname{Re} H^{1}$ such that

$$
\left\|T_{k} f\right\|_{\operatorname{Re} H^{1}} \leq\|f\|_{k}, \quad f \in C_{k} .
$$

3. Operator $T_{k}$ as a multiplier. For any integer $n$ and $t$ in $T$ we put $e_{n}(t)=\exp (2 \pi i n t)$. A direct calculation of the value of operator $T_{k}$ on $e_{n}$ leads to the following theorem.

THEOREM 3. Operator $T_{k}$ is of multiplier type, i.e. $T_{k} e_{n}=\beta_{n}(k) e_{n}$, $n \in Z$, where $\beta_{n}(k)$ is given by the formula

$$
\beta_{n}(k)=\frac{1}{2 \pi^{2} n^{2}} \int_{(0,1]}\left(\cos (2 \pi n s)-1+2 \pi^{2} n^{2} s^{2}\right) \frac{1}{s^{3}} d \mu(s), \quad n \neq 0 .
$$

Moreover, one has $\beta_{0}(k)=0$ and $\beta_{n}(k)=\beta_{-n}(k)>0$ for $n \neq 0$, and $T_{k}$, restricted to $C_{k}(0)=\left\{f \in C_{k} ; \int_{T} f(t) d t=0\right\}$, is injective.

It is a well-known result of Hardy [3] that if $\sum_{n} c_{n} e_{n}$ is the Fourier series of a function $g \in \operatorname{Re} H^{1}$ then $\sum_{n=0}\left|c_{n} / n\right|<C\|g\|_{\operatorname{Re} H^{1}}$ for certain constant $C$ independent of $g$. This fact together with Theorems 1 and 2 imply the following corollary. 
COROLLARY. Let $f$ be a continuous function of bounded k-entropy. If $\sum_{n} a_{n} e_{n}(t)$ is the Fourier series of $f$, then $\sum_{n \neq 0} \beta_{n}(k)\left|a_{n} / n\right| \leq C\|f\|_{k}$.

EXAMPLES. 1. If $k(s)=s$, then $\mu$ is the point mass measure $\delta_{1}$ concentrated at $s=1$ and $\beta_{n}(k)=1$ for any $n \neq 0$. Note that in this case $\|f\|_{k}$ is just the oscillation of a function $f$ on $T$.

2. Suppose that $d \mu(s)=q s^{q-1} d s$ for some $q, 0<q \leq 1$. Then a repeated integration by parts leads to the formula

$$
\begin{aligned}
\beta_{n}(k) & =\frac{q}{(q-2)(q-3)} \\
& \times\left(q-4+2 n^{1-q} \int_{0}^{n}(1-\cos (2 \pi y)) y^{q-2} d y\right), \quad n>0 .
\end{aligned}
$$

In particular, if $k(s)=s(|\log (s)|+1)$ is the Shannon entropy, then $d \mu(s)=$ $d s$ (see [2]) and $\beta_{k}(n)=\int_{0}^{n}(1-\cos (2 \pi y)) y^{-1} d y-(3 / 2), n>0$. Now it is not difficult to prove that $\lim _{n \rightarrow 0}\left(\beta_{n}(k) / \log (n)\right)=1$. Therefore, using Theorem 3 we conclude that if $\sum_{n} a_{n} e_{n}$ is the Fourier series of a continuous function of bounded Shannon entropy norm then $\sum_{n \neq 0} \mid a_{n}(\log (|n|) / n \mid<\infty$. Similarly, formula (3.2) implies that if $\left\{a_{n}\right\}_{n \in Z}$ are the Fourier coefficients of a continuous function with bounded Lipschitz entropy norm (i.e. $k(s)=s^{q}, 0<$ $q<1$, and by [2] $\left.d \mu(s)=q d \delta_{1}(s)+q(q-1) s^{q-1} d s\right)$, then $\sum_{n \neq 0}\left|a_{n}\right| /|n|^{q}<\infty$.

\section{REFERENCES}

1. R. R. Coifman, A real variable characterization of $H^{p}$, Studia Math. 51 (1974), 269274.

2. R. Dabrowski, Probability measure representation of norms associated with the notion of entropy, Proc. Amer. Math. Soc. 90 (1984), 263-268.

3. P. Duren, Theory of $H^{p}$ spaces, Academic Press, New York, 1970.

4. P. Koosis, Introduction to $H^{p}$ spaces, London Math. Soc. Lecture Note Series 40, Cambridge Univ. Press, Cambridge and New York, 1980.

5. B. Korenblum, On a class of Banach spaces associated with the notion of entropy, Trans. Amer. Math. Soc. 290 (1985), 527-553.

6. _ A generalization of two classical convergence tests for Fourier series and some new Banach spaces of functions, Bull. Amer. Math. Soc. (N.S.) 9 (1983), 215-218.

7. K. Yosida, Functional analysis, Grundlehren Math. Wiss. Band 123, Springer-Verlag, Berlin and New York, 1974, pp. 130-136.

Department of Mathematics, Columbia University, New York, New YORK 10027 